Sonderdruck aus:
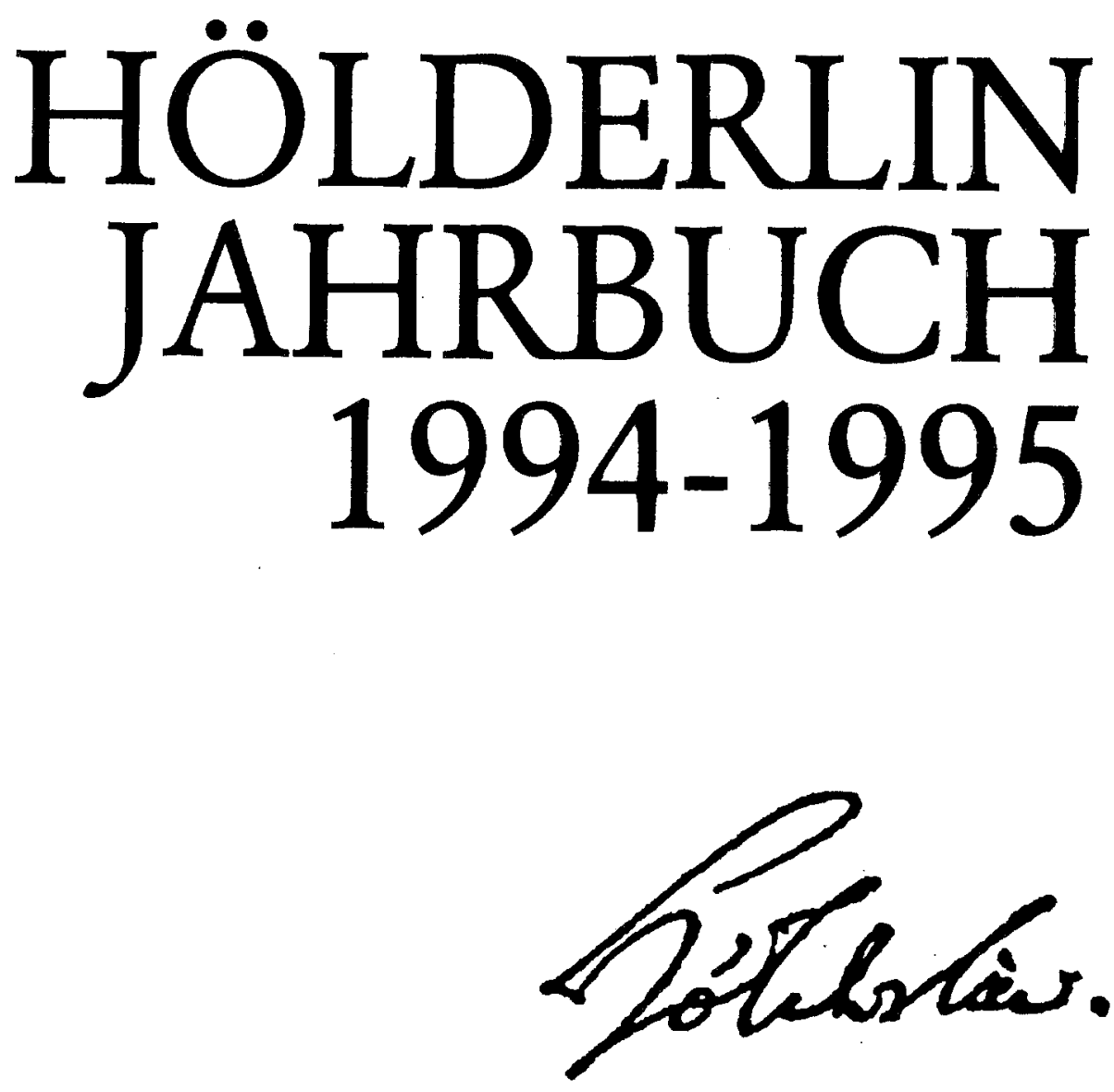

J.B. METZLER 


\section{Bemerkungen bezüglich der Rezeption Hölderlins in Spanien}

Von

Anacleto Ferrer

Wenn die Literatur nationale Grenzen überschreitet, geraten das Werk und sein Autor in Kontakt mit einigen Lesern, deren Mentalität und Vorliebe sich manchmal sehr von denen seines ursprünglichen Publikums entfernt. Die literarischen Qualitäten und der schöpferische Inhalt erleiden das äußerliche Auf und $\mathrm{Ab}$ der Kulturpolitik, der Ökonomie und der Ideologie des Rezeptionslandes. Hölderlin und sein Werk bilden keine Ausnahme von diesem grundlegenden Prinzip der Literatursoziologie. Die Geschichte der Rezeption Hölderlins in Spanien ist lang - sie beginnt mit dem Jahr seines Todes - und voller Wagnis, von Glücksfällen heimgesucht; bis heute existiert keine Chronologie, die absolut zuverlässig wäre. Auf diesen Seiten wollen wir dazu beitragen, einen historischen Rahmen der Rezeption zu schaffen und einige Zweifel über jene Rezeption aufzuklären.

\section{Die erste Nachricht über Hölderlin in Spanien}

Am 14. März 1843, fast drei Monate vor dem tatsächlichen Ereignis, gab 'El Heraldo. Politische, religiöse, literarische und industrielle Zeitung', in der Literaturabteilung der Nummer 204 den Tod Hölderlins bekannt. Der Artikel im 'El Heraldo' ist nicht unterzeichnet und, außer den wenigen Zeilen, die wir jetzt zitieren werden, auch kein Original:

Die Geschichte dieses Dichters [...] läßt sich in zwei Worten zusammenfassen: Hochmut und Verfall. Hölderlin verkörperte jenen Typus von Schwärmerei, Menschenhaß und Wahnsinn, der das schöne Mode-Ideal ist, in dem sich so viele verirte jugendliche Köpfe gefallen und der bedauerlicherweise mit der wahren Seelengröße und dem Leben der Poesie verwechselt wird. Aber in Hölderlin hat man auch das warnende Beispiel vor sich, und gewiß ist die Warnung selten handgreiflicher und schrecklicher gewesen: Nachdem er in wenigen Jahren sein Empfindungsvermögen sozusagen vergeudet und sein Leben verschleudert hatte, verfiel der Unglückliche in geistige Umnachtung.

(StA VII 4, 222) 
Der bedeutendste Teil des Textes besteht aus einer größtenteils wörtlichen Übersetzung einer anderen Schrift, die unter demselben Titel (HOELDERLIN) im 'Moniteur universel' am 8. Februar 1843 in Paris veröffentlicht wurde. J. Duesberg ist der Name des Autors. Aber auch dieser Artikel ist im ganzen kein Original; eine Reihe seiner Paragraphen bildet eine Mischung von biographischen Daten, viele von ihnen fehlerhaft, und Werturteilen bezüglich der Ursachen des Wahnsinns Hölderlins, die Duesberg dem Buch 'Friedrich Hölderlins Leben, Dichtung und Wahnsinn' von Wilhelm Waiblinger entnommen und übersetzt hat und an die er angeknüpft hat.

Der anonyme Artikelschreiber des 'El Heraldo' benutzt die Gelegenheit, die ihm der vermeintliche Tod des wandernden und ,in geistige Umnachtung" verfallenen Schriftstellers bietet, um die Rivalen-des reinsten Vertreters der liberalen Romantik José de Espronceda (1808-1842) heimtückisch zu verdammen. Die Geschichte ist bis zu diesem Punkt bekannt und befindet sich im Band VII4 (Rezensionen und Würdigungen 1791-1847) der Grossen Stuttgarter Ausgabe von Adolf Beck wiedergegeben. Jedoch möchten wir die von Beck angebotene Information mit einer Hypothese über den ,anonymen Artikelschreiber", der den Spaniern im vergangenen Jahrhundert den Namen Hölderlins zur Kenntnis brachte, sowie mit einer Beschreibung des ideologischen Charakters der Zeitung, die die falsche Nachricht vom Tode des Dichters verbreitete, ergänzen. Kaum dringen wir in das Geflecht der Geschichte ein, zeigt sich das Vorhandensein ideologischer Interessen, die den literarischen Werten des Werkes Hölderlins völlig fremd sind.

Am 16. Juni 1842 erschien die erste Nummer des 'El Heraldo', von Luís de Sartorius geleitet und seit seinem freiwilligen Exil in Paris von Donoso Cortés inspiriert. Diese Tageszeitung leitete die Schar der konservativen Blätter in offener Opposition gegen Espartero, einen progressiven General, der - angesichts der Minderjährigkeit Elisabeths der Zweiten die Regentschaft übernahm, nachdem die Mutter Elisabeths, Maria Cristina, am 12. Oktober 1840 gezwungenermaßen abgedankt hatte.

Nach ihrer Abdankung ging Maria Cristina nach Paris ins Exil, von einer zahlreichen Gruppe von Treuen begleitet, unter denen sich der berühmte und kompromißlos konservative Journalist Donoso befand. Aus diesem Kreise entsprang die Idee (und auch das Anfangskapital), eine Zeitung zu gründen, die seitens konservativer und Maria Cristina nahestehender Positionen Espartero zusetzen sollte. Donoso, der über gute Kontakte zu der konservativen Presse in Madrid verfügte, verwirklichte 
die Operation und beauftragte Luís de Sartorius und Ríos Rosas, beide zu der Zeit in Madrid lebend, mit der Angelegenheit. Ersterer sollte aus juristischen Gründen als Eigentümer der Zeitung auftreten.

Am 25. Juni 1842 schickt Donoso einen Brief an Sartorius und Ríos, in welchem sich eine 'Verordnung' für den 'El Heraldo' befindet. Nach der Weisung Donosos ,soll die Redaktion über eine politische Abteilung verfügen“, ferner „eine der Religion geweiht", „eine der Verwaltung und den materiellen Reformen geweiht" und eine letzte Abteilung, die sich der Literatur widmet und die ,in zwei Teile gegliedert ist: ein Teil weiht sich den dichterischen Originalkompositionen und der andere der Kritik unserer Schriftsteller, welcher Art sie auch seien"..

Die literarische Abteilung übernimmt Ríos Rosas, der am 30. Juli einen Brief von Donoso erhält, in dem dieser ihm sagt:

Ich bin davon überzeugh, daß die Zeitung bisher über keinen Literaturteil verfügen konnte, so wie ich davon überzeugt bin, daB Sie alles Mögliche tun werden, damit sie in Zukunft über einen solchen verfügen kann. Aber lassen Sie nicht $\mathrm{zu}$, daB diejenigen, die ihn schreiben werden, willkürlich vorgehen: zwischen der Literatur und der Politik befindet sich eine gewisse Verbindung. [...] Die Zeitung braucht Tatsachen, Klatsch usw., usw., und gerade das ist es, was ich nicht bieten kann, denn ich lebe zurückgezogen und ohne jeden Umgang mit der Welt. Deswegen habe ich mich schließlich dazu entschlossen, Grimaldi mit dem Schreiben zu beauftragen. Er spaziert den ganzen Tag lang über jene Straßen und weiß, was man sagt und tut, oder er erfindet es, und das ist genau, was die Zeitung braucht. ${ }^{2}$

Juan de Grimaldi war ein Franzose italienischer Herkunft, der 1823 mit den Truppen von Angoulême nach Spanien kam. Er ließ sich nieder, übersetzte Theaterstücke aus dem Französischen, heiratete die Schauspielerin Concepción Rodríguez und wurde Impresario des Prinzentheaters. Es ist sehr wahrscheinlich, daß Donoso, der wie Grimaldi mit dem literarischen Milieu Madrids verbunden war, ihn schon vor seiner Ausreise nach Frankreich kannte.

Am 5. November brechen Donoso und Ríos ihre Beziehungen zu Sartorius ab. Das Motiv: eine nicht sehr klare Handhabung des Geldes, das man ihm für die Zeitung aus der französischen Hauptstadt zukommen

\footnotetext{
' Zitiert nach Federico Suárez, Donoso Cortés y la fundación de El Heraldo y El Sol, Pamplona 1986, 220-221.

${ }^{2}$ Ebd., 231-232.
} 
ließ. Aber Sartorius ist der rechtmäßige Eigentümer der Firma und fuihrt seit diesem Datum den 'El Heraldo' alleine. Grimaldi wird dem 'El Heraldo' weiterhin seine Chroniken aus Paris schicken, so sehr Donoso auch versucht hat, ihn zu einem Bruch mit Sartorius zu bewegen. Und wer, wenn nicht Grimaldi, konnte den ins Kastilische übersetzten und mit Hintergedanken ergänzten Artikel Duesbergs, der anfangs Februar 1843 im 'Moniteur universel' erschienen war, in einem Moment senden, in dem die Beziehungen zwischen 'El Heraldo' und den spanischen Konservativen im Exil abgebrochen waren?

Erinnern wir uns an die Ermahnung, die Donoso Cortés Ríos Rosas gab: „Zwischen der Literatur und der Politik befindet sich eine gewisse Verbindung". Das Beispiel Hölderlins sollte den jungen liberalen spanischen Dichtern als Warnung dienen. Sein Name erscheint zum ersten Male in Spanien mit einer Zeitung verknüpft, die das Herzstiuck der konservativen Strategie der Hetze und des Umsturzes der Espartero nahestehenden und Maria Cristina feindlich gesinnten Liberalen bildete.

\section{Die erste Übersetzung Hölderlins in Spanien}

Auch die erste Übersetzung Hölderlins in Spanien findet sich von einem mysteriösen Schein umgeben: Welches Datum trägt die erste Übersetzung? Wer ist der Autor? In welche Sprache wurde zuerst übersetzt? Ins Katalanische oder ins Kastilische?

Jaume Medina, der Autor der bemerkenswerten Arbeit über 'Carles Riba i Hölderlin', die den ersten ernsthaften Versuch darstellt, die Rezeption Hölderlins in eine der vier gesprochenen Sprachen in Spanien zu untersuchen, hält den katalanischen Dichter für den „wahrhaftigen Einführer Hölderlins in die katalanische (und wenn man so will, auch in die kastilische) Literatur “. ${ }^{3}$ Diese Bemerkung ist nicht exakt: Riba ist der erste, der Hölderlin ins Katalanische übersetzt hat, aber nicht ins Kastilische. Als Riba seine ersten Übersetzungen ins Katalanische veröffentlichte, gab es schon - wie wir sehen werden - eine Übersetzung ins Kastilische aus der Feder eines anderen berühmten Katalanen, Manuel de Montoliu.

Im März 1922 reist der Hellenist und Dichter Carles Riba mit einem von der Mancomunitat de Catalunya gewährten Stipendium nach

\footnotetext{
${ }^{3}$ Jaume Medina, Carles Riba i Friedrich Hölderlin, Barcelona 1987, 24.
} 
München, um unter der Leitung des Philologen Karl Vossler zu studieren. Am 3. Juli 1922 schreibt er aus München an seinen Freund López-Picó, um ihm eine erfreuliche Nachricht mitzuteilen: „Ich habe (was natürlich uns anbelangt) einen großartigen lyrischen Dichter entdeckt, einen Zeitgenossen Goethes, der uns manchmal gleicht: Hölderlin “. ${ }^{4}$ Die Entdeckung Hölderlins seitens Riba offenbart sich dußerlich in der Form seiner Fassung der 'Set poemes de Hölderlin', die in der Ausschreibung der Juegos Florales del Ampurdán, einem literarischen Wettbewerb, von 1922 vorgestellt wurde. Im Jahr 1923 verlegte Publicacions Empordá in Barcelona einen Band mit den Fassungen Ribas.

$\mathrm{DaB}$ Manuel de Montoliu anfangs der zwanziger Jahre das Werk Hölderlins kennt und daß er für einen Verlag einige seiner Gedichte in einer Anthologie kompiliert und übersetzt, in der das Erscheinungsjahr nicht angegeben ist, ist auch Medina bekannt. ${ }^{5}$ Ein Besuch in der Nationalbibliothek in Madrid gab uns das Datum bekannt, an dem der Band XXX der Sammlung 'Die besten (lyrischen) Gedichte der besten Dichter' des Cervantes Verlags veröffentlicht wurde. Dieser Band ist Hölderlin gewidmet und enthält zwanzig von Manuel de Montoliu übersetzte Gedichte: das Buch wurde im Jahr 1921 veröffentlicht, ein Jahr bevor Riba Hölderlin ,entdeckt“ und zwei Jahre bevor seine ersten Fassungen erscheinen. Dies wird ersichtlich aus der Referenznummer 38.745 des dritten Bandes (Buchstaben H/M) des 'Generalkatalogs der spanischen und lateinamerikanischen Büchersammlungen', der von Bibliothekaren der Nationalbibliothek angefertigt wurde und die Jahre zwischen 1901 und 1930 umfaßt.

Im Jahr 1908 gewärte der Provinzialrat von Barcelona Manuel de Montoliu ein Stipendium, um bei den Professoren Schädel und Suchier Romanistik in Halle zu studieren, wo er sich drei Jahre lang aufhielt. 1920 kehrte er dieses Mal nach Hamburg zurück, wo er als kastilischer und katalanischer Lektor tätig war. Konnte er es vielleicht nicht sein, der in seinen beiden Aufenthalten Hölderlin ,entdeckte“?

Falls wir recht haben, wurde Hölderlin in den frühen zwanziger Jahren durch Katalonien in Spanien eingeführt, sowohl ins Kastilische als auch ins Katalanische: Manuel de Montoliu war der erste, der Hölderlin in eine der vier spanischen Sprachen übersetzt hatte, Carlos Riba der zweite.

\footnotetext{
${ }^{4}$ Zitiert nach ebd., $12 \mathrm{f}$.

${ }^{5}$ Ebd., 95.
} 
Von den dreißiger Jahren bis anfangs der siebziger Jahre

Zwischen 1922 und 1944 arbeitet Riba weiterhin an seinen Übersetzungen Hölderlins ins Katalanische, bis er im ganzen vierundzwanzig Gedichte vereinigt hat. Die erste Ausgabe seiner 'Versions de Hölderlin' trägt das Jahr 1943 in Buenos Aires, aber in Wirklichkeit wurden 1944 in Barcelona 125 Exemplare unerlaubt veröffentlicht.

In den folgenden Jahren erscheinen mehrere Hölderlinübersetzungen; es handelt sich immer um kurze Anthologien und einzelne Gedichte in Literaturzeitschriften, die jeweils an ein geringes Publikum gerichtet waren. Im Jahr 1935 übersetzt Luís Cernuda mit der Hilfe Hans Gebsers, eines Hispanisten und Autors eines Buches über Rilke in Spanien, einige Gedichte Hölderlins, die in der Zeitschrift 'Cruz y Raya' veröffentlicht werden. José María Valverde, ebenfalls ein vortrefflicher Hölderlinübersetzer, kommentiert die außerordentliche Wirkung, die die Hölderlinübersetzung in der Lyrik Cernudas hervorgerufen hat: „Man vernimmt seinen Widerhall, besonders in 'Invocaciones a las gracias del mundo', in hervorragender Harmonie mit dem Widerhall der englischen Romantiker und mit denen gewisser lateinischer Elegiendichter. Und eines Tages kann man vielleicht erwägen, ob diese Welle über Cernuda auch Vicente Aleixandres 'Sombra del Paraíso' erreicht hat. Auf alle Fälle geschah es unter dem lyrischen Pontifikat Vicente Aleixandres, als Hölderlin begann, sich im Land gleich wie in seinem Hause zu bewegen". 6 Diese Bemerkung Valverdes ist sehr anregend, aber es ist nicht unsere Absicht, jenes auszuwerten, was wir innere Rezeption Hölderlins nennen könnten jenen Widerhall, den das aufmerksame Lesen eines anderen Dichters in einem Dichter hervorruft -, sei es über Cernudas 'Invocaciones' oder über die erste Ausgabe des 'Ocnos', sei es über Ribas zweites Buch 'Estances' oder über die 'Elegies de Bierville'. Valverde, der im Jahr 1949 zwölf Gedichte veröffentlicht hatte, erinnert sich an seine eigene Erfahrung als Hölderlinübersetzer: „Ich habe mich auf die Übersetzung Hölderlins gestürzt und so meine Beschäftigung mit Rilke - von 1943 bis 1944 - verlängert. [...] Als Dichter, der damals den Grad des Lizenziats in Philosophie erwarb und der sich als Übersetzer versuchte, verstand ich Hölderlin schon nicht mehr als Anreiz, sondern als eine Herausforderung. Und vielleicht geschah meine Annäherung nicht nur allein aus persön-

\footnotetext{
' José María Valverde in der Einfuhrung zu seiner Ubersetzung der 'Poemas' Friedrich Hölderlins, Barcelona 1983, 14.
} 
lichen Gründen heraus, sondern auch im Namen meiner Umgebung und dessen, was wir damals Generation nannten; in günstigen Umständen, die ich damals vorübergehend genoB: der Name Hölderlins ließ jedes lyrische und philosophische Ohr aufhorchen".? In der Tat, im Spanien jener Jahre, jener letzten Jahre des Zweiten Weltkrieges, befand sich Hölderlin unter den wenigen benutzbaren Resten des deutschen Propagandaeinflusses, von politischen Resonanzen befreit und durch die Essays von Dilthey in 'Das Erlebnis und die Dichtung' und von Heidegger in 'Hölderlin und das Wesen der Dichtung' gegenwärtig, die auch in den vierziger Jahren dem spanischen Leser bekannt gemacht wurden - der erstere durch den mexikanischen Verlag Fondo de Cultura Económica, der zweite durch die Zeitschrift 'Escorial'.

Innerhalb der fünfziger Jahre und anfangs der siebziger ist das Werk Hölderlins Gegenstand zahlreicher Übersetzungen. Der Ton, der die Jahrzehnte der dreißiger und vierziger Jahre geprägt hatte, besteht weiterhin fort: das Fehlen einer systematischen Annäherung an das literarische Werk des Dichters, denn immer handelt es sich um kurze Anthologien, die nach der Vorliebe und den persönlichen Interessen des Kompilators angefertigt werden und deren nicht zahlreiche Ausgaben an ein gebildetes, aber geringes Publikum gerichtet sind. Die normalisierte Rezeption Hölderlins und die Vermehrung der Zahl seiner Leser beginnt erst seit 1976, zu Beginn des politischen Übergangs zur Demokratie in Spanien.

\section{Vom politischen Übergang in Spanien bis zu den heutigen Tagen}

Der gewaltige Versuch, die verlorenen Etappen in der langen Stagnation, die im Franquismus durch die Routine und die Zensur verursacht wurden, aufzuarbeiten, wurde seit 1976 mit dem Tode des Diktators und den ersten prädemokratischen Reformen von einem euphorischen Ansporn der Autoren begleitet, die in dem mehrere Jahrzehnte lang dauernden Regime verbannt und geächtet waren. Die demokratische Entwicklung sensibilisiert die jungen Leser, die Studenten, die kritischen Intellektuellen und die Verleger, die den Wahnsinn Hölderlins und seine glühenden Gesänge an die Freiheit zu einer Waffe im Kampf gegen jedwede Form von Autoritarismus machten. Die aufsteigende Kurve Hölderlins, die gegen Ende

\footnotetext{
${ }^{7}$ Ebd., $15 f$.
} 
der siebziger Jahre schwindelerregend ist, regt uns an, über die Umstände nachzudenken, unter denen die Rezeption des Werkes in diesen Jahren stattfindet.

In der einzigen veröffentlichten Arbeit über 'Die Rezeption der deutschen Romantik in Spanien in der politischen Übergangszeit' schreibt Manuel José González: „Die soziologische Forschung der Massenkommunikation bietet uns andere Regeln an, um die Zeichen und Indizien ausfindig zu machen, die das Medium Buch bezüglich der potentiellen Aufmerksamkeit der Leser oder bezüglich der Verbreitung eines Werkes, Autors oder einer Ideologie benutzt. Beispielhaft sind: der Prolog, die Vorbemerkung oder der Epilog, der Werbetext des Umschlags, die Widmung, usw. ${ }^{\text {“8 }}$ Wir werden jetzt den Klappentext der spanischen Fassung des 'Hyperion oder der Eremit in Griechenland' von Jesús Munárriz zitieren; ein Buch, das den wahrhaftigen Wendepunkt der Rezeption Hölderlins in Spanien darstellt:

Des Herzens Wooge schdumte nicht so schbin empor, und wirde Geist, wenn nicht der alte stumme Fels, das Schiksaal, ihr entgegenstande. (StA III, 41)

Immerhin hat das den Staat zur Hölle gemacht, daß ihn der Mensch zu seinem Himmel machen wollte. (StA III, 31)

Es werde von Grund aus anders! Aus der Wurzel der Menschheit sprosse die neue Welt! Eine neue Gottheit walte uber ihnen, eine neue Zukunft kldre vor innen sich auf. In der Werkstatt, in den Hausern, in den Versammlungen, in den Tempeln, uberall werd' es anders! (StA III, 89)

Dieses Buch - der erste Titel des Verlags, der seinen Namen trägt und der von seinem Übersetzer geleitet wird - verbindet sich mit den Erwartungen des politischen Wechsels und mit dem Wunsch nach Freiheit der spanischen Gesellschaft in jenen ersten prädemokratischen Jahren. Dergestalt hat das Werk Hölderlins eine Aufnahme im Publikum und eine Kritik erhalten, die jedwede vorherige Übersetzung des Dichters bei weitem übersteigt. 1992 - fünfzehn Jahre nach seiner Veröffentlichung erschien die dreizehnte Ausgabe des 'Hyperion', daß heißt, man hat mehr als 25.000 Exemplare des Buches verkauft, was für einen deutschen Klassiker in Spanien mehr als außergewöhnlich ist.

\footnotetext{
${ }^{8}$ Manuel José Gonzáles, La recepción del romanticismo alemán en la España de la transición (1970-1980). Filología Moderna, Universidad Complutense, Madrid 1982, $7-30 ; 13 \mathrm{f}$.
} 
Jener Hölderlin, der 1843 den Verlegern des 'El Heraldo' als Waffe gegen die jungen liberalen Dichter gedient hatte, wird nun in einem Spanien, das um die Wiedergewinnung seiner demokratischen Würde kämpft, die ihm ein halbes Jahrhundert Diktatur usurpiert hatte, als Vorkämpfer der Freiheit begrüBt. Die Geschichte schließt sich über sich selbst. Vom Verlegererfolg des 'Hyperion' unterstützt, wurden die früheren Übersetzungen neu herausgegeben - alle, mit der erstaunlichen Ausnahme der ersten, aus der Feder Manuels de Montoliu stammend, die anscheinend fast niemand kennt; so erschien im Jahr 1979 von neuem die Übersetzung des 'Archipelagus', die Manuel Díez del Corral 1941 für die Zeitschrift 'Escorial' angefertigt hat - eine Veröffentlichung in enger Verbindung mit der spanischen Falange. Der Alianza-Verlag, der aktuelle Verleger, benutzt den Erfolg des Romans, um seinen Autor auf der Rückseite vorzustellen:

Auch wenn man im XIX. Jahrhundert Friedrich Hölderlin für einen schwachen und nostalgischen Dichter gehalten hat, hat der Zug der Zeit das Irrtümliche dieser Interpretation bewiesen; sie hat den Autor des 'Hyperion' auf einen der Gipfel der Universalliteratur erhöht und hat ihn zu einem Vorläufer und Meister der Sensibilität unserer Zeit geweiht.

Heute, 150 Jahre nach dem Tode des Dichters und der ersten Veröffentlichung seines Namens in Spanien, ist Hölderlin ein Autor, dessen Werk fast vollständig übersetzt, umfangreich neu herausgegeben ist und über ein treues Publikum verfügt. Man hat begonnen, einige klassische Studien über sein Werk, wie die von Bertaux oder Szondi, zu übersetzen, und die Bücher spanischer Schriftsteller, die Aspekte seiner Lyrik oder seiner Philosophie berühren, werden immer zahlreicher.

Weder Schiller noch Kleist oder Novalis - um nur einige der zeitgenössischen umfangreich übersetzten Schriftsteller zu nennen - genießen in Spanien dieses Wohlgefallen der Verleger und diese Anerkennung, die man Hölderlin zukommen läßt. Jedes Jahr erscheinen neue Übersetzungen, und es scheint, daß das Werk Hölderlins sich endgültig von dem Auf und $\mathrm{Ab}$ der Kulturpolitik, der Ökonomie und der Ideologie, von denen wir in den ersten Zeilen dieser Arbeit gesprochen haben und die seine Rezeption bis vor kurzem geprägt haben, gelöst hat. 